\title{
The class of 2-dimensional neat reducts is not elementary
}

\author{
by \\ Tarek Sayed Ahmed (Giza)
}

\begin{abstract}
S C, C A, Q A$ and $Q E A$ stand for the classes of Pinter's substitution algebras, Tarski's cylindric algebras, Halmos' quasipolyadic algebras and Halmos' quasipolyadic algebras with equality, respectively. Generalizing a result of Andréka and Németi on cylindric algebras, we show that for $K \in\{S C, Q A, C A, Q E A\}$ and any $\beta>2$ the class of 2 -dimensional neat reducts of $\beta$-dimensional algebras in $K$ is not closed under forming elementary subalgebras, hence is not elementary. Whether this result extends to higher dimensions is open.
\end{abstract}

0. Introduction. Neat reducts and related notions like neat embeddings play a central role in Algebraic Logic. One of the main representation methods used in the theory of cylindric algebras and variants thereof like polyadic algebras is based on the Neat Embedding Theorem, to be recalled below. (See also [15, p. 400] and [16, Thm. 3.2.10]). It is known (cf. [29] or [31]) that the Neat Embedding Theorem, or NET for short, proved by Henkin in the fifties is an algebraization of Henkin's celebrated proof of the completeness of first order logic. Ever since, variants of the NET have been successfully applied to (algebraically) prove completeness of other versions of quantifier logics (e.g. Keisler's logics, cf. [12]). Other works adressing the notion of neat reducts, in one way or another, include [1], [29] and [27]. In [1] and [29], neat reducts are studied in connection to (isomorphism types of) algebras of sentences of first order logic. In [27] a NET is formulated and proved which implies the completeness of certain finitary fragments of Keisler's logics that are also expansions of first order logic without equality. This provides a solution to the so-called finitization problem in Algebraic Logic. In fact, the NET has proved to be a successful strategy to address different versions of the finitization problem (see e.g. [35]). Very

2000 Mathematics Subject Classification: Primary 03G15; Secondary 06E25, 08B99.

Key words and phrases: Algebraic Logic, cylindric algebras, quasipolyadic algebras, neat reducts, elementary.

Research was supported by the Hungarian National Foundation for Scientific Research OKTA grant no T30314. 
roughly the finitization problem is: Find a structural (in the sense of [10]) extension or modification of first order logic that admits a strongly complete finite Hilbert-style axiomatization in the sense of [6]. The finitization problem, traced back at least to the works of Alfred Tarski on relation algebras in the forties (cf. [36]), is currently a very active and rich research direction in Algebraic Logic (cf. [24, the subsections on finitization], [27], [30] and [26]). The connection of neat reducts to other metalogical properties like the Beth-definability property and omitting types can be found in [30]. This (and other) connection(s) are investigated more thoroughly in [25], [20], [21], [23], [13], [14], [5], [8], [31] and [34]. We should point out that this list of references addressing the important notion of neat reducts in Algebraic Logic is far from being complete.

History (Previous results). Let $\alpha<\beta$ be ordinals. Solving problem 2.11 of [15], Németi proves in [23] that the class $\mathbf{N} r_{\alpha} C A_{\beta}$, of $\alpha$-dimensional neat reducts of $\beta$-dimensional cylindric algebras, though closed under homomorphic images and products, is not a variety (i.e. it is not closed under forming subalgebras). Independently, Maddux [21] obtained a partial solution of problem 2.11 of [15] proving that $\mathbf{N} r_{3} C A_{\beta}$ is not closed under forming subalgebras for $\beta \geq 5$. The significance of the closure of the class of neat reducts under forming subalgebras in connection to proving amalgamation results was discovered by Pigozzi [25, Lemma 2.2.12], and this tie is further emphasized and pursued in [34] and [31]. In [34] Németi's result - concerning closure of the class of neat reducts under forming subalgebras - is generalized to other classes frequently studied in Algebraic Logic. These include Halmos' quasipolyadic algebras and Pinter's substitution algebras and various reducts thereof. In particular, it is shown in [34] that for $K \in\{S C, Q A, Q E A\}$, the class $\mathbf{N} r_{\alpha} K_{\beta}$ of $\alpha$-dimensional neat reducts of $\beta$-dimensional algebras in $K$ is not closed under forming subalgebras for $\alpha \geq 2$ and $\beta>\alpha$. In particular, this class - though closed under homomorphic images and products and hence under ultraproducts $\left(^{1}\right)$ — cannot be axiomatized by any set of equations (cf. [34]). Motivated to give some (hopefully first order) characterization of such classes, it is thus natural to ask whether the class of neat reducts is perhaps closed under forming elementary subalgebras, so that it is an elementary (i.e. first order axiomatizable) class. In [17, II.8.6, p. 266], Andréka and Németi provide a negative answer for the cylindric case but only for the lowest value of $\alpha$. They show that the class $\mathbf{N} r_{2} C A_{\beta}$ for $\beta>2$ is not even closed under forming elementary subalgebras. That $\mathbf{N} r_{1} C A_{\beta}$ is a variety is proved by Németi in [23]. $\mathbf{N} r_{0} C A_{\beta}$ is just the class of boolean algebras and thus is also a variety. In [30] this result is generalized to higher dimensions, but only for cylindric

$\left({ }^{1}\right)$ Recall that an ultraproduct is a homomorphic image of a product. 
algebras. Thus for any pair of ordinals $1<\alpha<\beta$ the class $\mathbf{N} r_{\alpha} C A_{\beta}$ is not closed under forming elementary subalgebras, hence this class is not elementary. This confirms a conjecture of Németi formulated in [23] and solves problem 4.4 of [16]. Here, as indicated in the abstract, we extend this result to Halmos' quasipolyadic algebras and Pinter's substitution algebras, but again only for the lowest values of $\alpha$, namely when $\alpha=2$. It seems that the generalization of this result to higher dimensions is not trivial. We conjecture, though, that it can proved - using a combination of the ideas presented herein with those in [30] - that for any pair of ordinals $2<\alpha<\beta$ the class $\mathbf{N} r_{\alpha} K_{\beta}$ is not first order axiomatizable. As it is closed under ultraproducts, this amounts to showing that it is not closed under elementary equivalence or equivalently - by the celebrated Keisler-Shelah ultrapower theorem (cf. [11, Theorem 6.15])-under ultraroots.

Organization. In Section 0, we review the basic notions and formulate the main theorems. The proofs are given in Section 1.

0. Basic notions and main results. To formulate our results we review some basic notions from [16] and [24]. We start by defining the algebras we shall be dealing with. From now on, $K \in\{S C, C A, Q A, Q E A\}$, where $S C, C A, Q A$ and $Q E A$ abbreviate the classes of substitution, cylindric, quasipolyadic, and quasipolyadic equality algebras, respectively. $K_{\alpha}, \alpha$ an ordinal, stands for the class of all algebras in $K$ of dimension $\alpha$, sometimes referred to as $\alpha$-dimensional algebras. Algebras will be denoted by calligraphic letters, and when we write $\mathcal{A}$ then we will be tacitly assuming that $A$ will denote the universe of $\mathcal{A}$. Let $\mathcal{A} \in K_{\alpha}$. Then $\mathcal{A}$ is a boolean algebra with extra operations. In fact, $\mathcal{A}$ will be a boolean algebra with operators. In particular, the non-boolean extra operations are required to distribute over the boolean join. The basic boolean operations of $\mathcal{A}$ will be denoted by $\cup, \cap, \backslash, 0,1$, standing for join, meet, complementation, least and greatest elements, respectively.

An algebra in $S C_{\alpha}$ is of the form $\mathcal{A}=\left\langle A, \cup, \cap, \backslash, 0,1, c_{i}, s_{i}^{j}\right\rangle_{i, j \in \alpha}$ where

(1) $\langle A, \cup, \cap, \backslash, 0,1\rangle$ is a boolean algebra $(B A)$, hereafter denoted by $\mathrm{Bl} \mathcal{A}$.

(2) $c_{i}$ and $s_{i}^{j}(i, j \in \alpha)$ are unary operations on $A$ satisfying a finite schema of equations. As we shall be mostly dealing with the concrete versions of $S C_{\alpha}$ 's, the so-called representable ones, we do not have to remember all those schemas; we recall from [24] only those we need.

Proposition 0.1. Let $\mathcal{A} \in S C_{\alpha}$. Then the following equations hold for any $i, j, k \in \alpha$ and any $x, y \in A$ :

$\left(E_{1}\right) \quad c_{j} 0=0, x \leq c_{i} x, c_{i}\left(x \cap c_{i} y\right)=c_{i} x \cap c_{i} y$, and $c_{i} c_{j} x=c_{j} c_{i} x$. 
In other words the $c_{i}$ 's are complemented closure operators and $c_{i}, c_{j}$ commute.

$\left(E_{2}\right) \quad s_{i}^{i} x=x$.

$\left(E_{3}\right) \quad s_{j}^{i}$ are $B A$ endomorphisms.

$\left(E_{4}\right) \quad s_{j}^{i} c_{i} x=c_{i} x$.

$\left(E_{5}\right) \quad c_{i} s_{j}^{i} x=s_{j}^{i} x$ whenever $i \neq j$.

$\left(E_{6}\right) \quad s_{j}^{i} c_{k} x=c_{k} s_{j}^{i} x$ whenever $k \notin\{i, j\}$.

$\left(E_{7}\right) \quad c_{i} s_{i}^{j} x=c_{j} s_{j}^{i} x$.

$\left(E_{8}\right) \quad s_{j}^{i} s_{i}^{k} c_{i} x=s_{j}^{k} c_{i} x$.

In our treatment of $C A_{\alpha}$ 's, we follow the monographs [15], [16], while in our treatment of $Q A_{\alpha}$ 's and $Q E A_{\alpha}$ 's, we rather follow [28]. In more detail, we think of $Q A_{\alpha}$ 's as algebras of the form $\left\langle\mathcal{A}, p_{i j}\right\rangle_{i, j \in \alpha}$, where $\mathcal{A} \in S C_{\alpha}$, and for all $i, j \in \alpha, p_{i j}$ are unary operations on $A$, which also happen to be boolean endomorphisms. $Q E A_{\alpha}$ 's, on the other hand, are expansions of $Q A_{\alpha}$ 's by diagonal elements $\left(d_{i j}, i, j \in \alpha\right)$. For an explicit finite schema of equations axiomatizing the variety $K_{\alpha}$, we refer the reader to the appendix of [24] $\left({ }^{2}\right)$. For the purposes of the present paper, however, it is, enough to know that all algebras considered are expansions of $S C_{\alpha}$ 's; that is, if $\mathcal{A} \in K_{\alpha}$, its $S C$ reduct obtained by deleting the $p_{i j}$ 's and $d_{i j}$ 's, if any, in symbols $\operatorname{Rd}_{S C} \mathcal{A}$, is (term equivalent to) an $S C_{\alpha}$. In particular, if $\mathcal{A} \in K_{\alpha}$, then $\operatorname{Rd}_{S C} \mathcal{A}=\left(E_{1}\right)-\left(E_{8}\right)$. Here by reduct we understand a generalized reduct. That is, the $S C$ operations may not be basic in the algebras considered, but are term definable. For example, in the standard formalism of $C A$ 's [15] and $Q E A$ 's [28], [24], $s_{j}^{i}$ is not a basic operation but is term definable by $s_{j}^{i}=c_{i}\left(x \cap d_{i j}\right)$. However when dealing with $Q E A^{\prime}$ 's, and for that matter with $C A^{\prime}$ 's, we shall often treat the term definable operation $s_{j}^{i}$ for all $i, j$, as a basic operation.

We now recall from [15, Def. 2.6.28] a notion that prevails throughout this paper, namely that of neat reducts.

Definition. 0.2. (i) Let $\mathcal{B} \in K_{\beta}$ and let $x \in B$. Then $\Delta x$, the dimension set of $x$, is defined to be the set $\left\{i \in \beta: c_{i} x \neq x\right\}$.

(ii) Let $\alpha<\beta$ be ordinals. Let $\mathcal{B} \in K_{\beta}$. Then the neat $\alpha$-reduct of $\mathcal{B}$, in symbols $\mathcal{N} r_{\alpha} \mathcal{B}$, is the $K_{\alpha}$ with universe $N r_{\alpha} B=\{b \in B: \Delta b \subseteq \alpha\}$, and whose operations are those of the similarity type of $K_{\alpha}$ restricted to $N r_{\alpha} B$.

$\left({ }^{2}\right)$ It is known that $\left\langle K_{\alpha}: \omega \leq \alpha\right\rangle$ forms a system of varieties definable by schemas, a concept originating with Monk from cylindric algebras, and further investigated in its own right by Andréka and Németi (cf. [16, pp. 260-263] for a sample of Andréka and Németi's results). 
It is not hard to see that $\mathcal{N} r_{\alpha} \mathcal{B}$ is closed under the indicated operations, so the definition of $\mathcal{N} r_{\alpha} \mathcal{B}$ is sound. It is also easy to see that $\mathcal{N} r_{\alpha} \mathcal{B} \in K_{\alpha}$. We should point out that neat reducts are subreducts (subalgebras of reducts) in the universal algebraic sense: when forming the neat $\alpha$-reduct of a $K_{\beta}$, we throw away not only operations, but also some elements of the algebra. We refer the reader to [7] where neat reducts were investigated in a (more general) "universal algebraic" setting.

Notation. For $L \subseteq K_{\beta}, \mathbf{N} r_{\alpha} L$ denotes the class of all neat $\alpha$-reducts of algebras in $L$. That is, $\mathbf{N} r_{\alpha} L=\left\{\mathcal{N} r_{\alpha} \mathcal{B}: \mathcal{B} \in L\right\}$.

It is known (see e.g. [15] or [29, Chapter 4] that $S \mathbf{N} r_{\alpha} K_{\beta}$ is a variety. Here $S$ stands for the operation of forming subalgebras. Borrowing terminology from [15, Remark 2.6.26], algebras in the class $S \mathbf{N} r_{\alpha} K_{\alpha+\omega}$ are said to have the neat embedding property, or $N E P$ for short. $\mathcal{A} \in K_{\alpha}$ has the NEP iff $\mathcal{A}$ is neatly embeddable in a $K_{\alpha+n}$ for every $n \in \omega$. A central theorem in the representation theory of such algebras, the so-called neat embedding theorem of [15], states that the class of algebras in $K_{\alpha}$ having the $N E P$ is precisely the class of representable $K_{\alpha}$ 's $\left(R K_{\alpha}\right)$. In symbols (see [16, Theorem 3.2.10], $[29$, Sec. 5.2])

$$
R K_{\alpha}=S \mathbf{N} r_{\alpha} K_{\alpha+\omega}=\bigcap_{n \in \omega} S \mathbf{N} r_{\alpha} K_{\alpha+n} .
$$

According to the conventions of the monograph [15], [16], an $R K_{\alpha}$ is a $K_{\alpha}$ that is isomorphic to a subdirect product of (weak) set algebras of dimension $\alpha$. To unify notation, we write $K s$ and $K s_{\alpha}$ to denote $K$ set algebras and $K$ set algebras of dimension $\alpha$, respectively. By the same token, $W K s_{\alpha}$ stands for weak $K$ set algebras of dimension $\alpha$, that is, $\mathcal{A} \in W K s_{\alpha}$ if $A$ is a boolean field of sets with greatest element (unit) a weak cartesian space, i.e. a set of the form

$$
{ }^{\alpha} U^{(p)}=\left\{s \in{ }^{\alpha} U:\left|\left\{i \in \alpha: s_{i} \neq p_{i}\right\}\right|<\omega\right\}
$$

for some $U$ and $p \in{ }^{\alpha} U$. In addition, $A$ of course has to be closed under the extra non-boolean $K_{\alpha}$ operations. For the sake of completeness, we recall from [24] the concrete interpretation of the non-boolean $Q E A_{\alpha}$ operations on $\alpha$-ary relations over $V \subseteq{ }^{\alpha} U$. Let $X \subseteq V$ and $i, j<\alpha$. Then

$$
\begin{aligned}
& c_{i}(X)=\left\{s \in V: \text { there exists } t \in X \text { such that } s_{j}=t_{j} \text { for all } j \neq i\right\}, \\
& s_{i}^{j}(X)=\{s \in V: s \circ[i \mid j] \in X\} .
\end{aligned}
$$

Here, $[i \mid j]$ denotes the transformation on $\alpha$ that sends $i$ to $j$ and is the identity on $\alpha \backslash\{i\}$. Next

$$
p_{i j}(X)=\{s \in V: s \circ[i, j] \in X\}
$$

where $[i, j]$ denotes the transposition on $\alpha$ that interchanges $i$ and $j$. And 
finally,

$$
d_{i j}=\left\{s \in V: s_{i}=s_{j}\right\} .
$$

Assume that $\mathcal{A} \in Q E A s_{2}$ with base $U$, i.e. $\mathcal{A}$ has greatest element $U \times U$. Then $p_{01} R$ for a binary relation $R \in A, R \subseteq U \times U$, is just forming the converse of $R$. $d_{01}$, on the other hand, is simply the identity relation on $U$. For their geometric meaning, $c_{i}$ is called the $i$ th cylindrification while $d_{i j}$ is called a diagonal element. $s_{i}^{j}\left(p_{i j}\right)$ is called the substitution operation corresponding to the transformation $[i \mid j]([i, j])$, or even simply a substitution. This stems rather from their metalogical interpretation, the latter being substitution of the variable $x_{i}$ for $x_{j}$ (interchanging the free occurrences of $x_{i}$ and $x_{j}$ ); cf. [24] or [29, Chapter 2], for further elaboration on such connections.

Unlike boolean algebras, not every $K_{\alpha}, \alpha>2$, is representable. In fact, the following is known. Let $\alpha<\beta$. Then $S \mathbf{N} r_{\alpha} K_{\beta}=K_{\alpha}=R K_{\alpha}$ if $\alpha \leq 1$. Here $S$ denotes the operation of forming subalgebras. For $\alpha>2$, the sequence $K_{\alpha} \supseteq S \mathbf{N} r_{\alpha} K_{\alpha+1} \supseteq S \mathbf{N} r_{\alpha} K_{\alpha+2} \supseteq \ldots$ is not eventually constant. This was proved and used by Monk for $C A$ 's to show that $R C A_{\alpha}$, for $\alpha>2$, is not finite schema axiomatizable $\left({ }^{3}\right)$. The analogous result for $S C$ 's, $Q A$ 's and $Q E A$ 's was proved by Johnson (the finite-dimensional case) [19], and Sain and Thompson (the infinite-dimensional case) [28]. When $\alpha=2$, then all diagonal free algebras, i.e. algebras in $S C_{2}$ and $Q A_{2}$, are representable (see [16, Theorem 5.4.33]). Though there are non-representable algebras in $K_{2}(K \in\{C A, Q E A\}), R K_{2}$ is finitely axiomatizable. Also $R K_{2}=S \mathbf{N} r_{2} K_{\beta}$ for all $\beta>2$. The latter two results are due to Henkin. The reader is referred to the comments in [16, below Theorem 4.1.44, pp. 125-126] for a fuller discussion of such results for $C A$ 's. The $S C, Q A$ and $Q E A$ analogues can be found in [3], [24] or/and [29, Chapter 4], and the references therein. We hasten to add that we shall be mostly dealing with (concrete) set algebras of dimension 2 . We are now ready to formulate the main results concerning $K_{\alpha}$ 's. Theorem 1 is proved in Section 1.

Theorem 1 (Main Theorem). Let $\beta>2$. Let $L$ be any class such that $W K s_{\beta} \subseteq L \subseteq K_{\beta}$. Then $\mathbf{N} r_{2} L$ is not closed under forming elementary subalgebras, hence is not elementary. In particular, the classes $\mathbf{N} r_{2} R K_{\beta}$ and $\mathbf{N} r_{2} K_{\beta}$ are not elementary.

1. Proofs. In this section we prove Theorem 1 . We construct an algebra $\mathcal{A} \in K s_{2} \cap \mathbf{N} r_{2} W K s_{\beta}$ for all $\beta>2$ and $\mathcal{B} \subseteq \mathcal{A}$ such that $\mathcal{B}$ is an elementary

$\left({ }^{3}\right)$ Lately Hirsch, Hodkinson and Maddux [18] proved that the above sequence for $\omega>\alpha>2$ is strictly decreasing, i.e. there is no $k \in \omega$ such that $\mathbf{S} N r_{\alpha} C A_{\alpha+k+1}=$ $S \mathbf{N} r_{\alpha} C A_{\alpha+k}$. This solves the long-standing open (neat embedding) problem 2.12 in [15]. It seems that their proof generalises to $S C$ 's, $Q A$ 's and $Q E A$ 's. The infinite analogue for $C A$ 's was settled by Pigozzi (unpublished). 
subalgebra of $\mathcal{A}$ and $\mathcal{B}$ is not (isomorphic to an algebra) in $\mathbf{N} r_{2} K_{\beta}$ for all $\beta>2$. We start off by fixing the notation.

Some notation. Our notation is mostly in conformity with that adopted in the monograph [15]. It is worthwhile though to review the notation mostly used.

For a set $X, \wp(X)$ denotes the set of all subsets of $X$, i.e. the powerset of $X$. Ordinals will be identified with the set of smaller ordinals. In particular, for finite $n$ we have $n=\{0, \ldots, n-1\}$. $\omega$ denotes the least infinite ordinal, which is the set of all finite ordinals.

${ }^{A} B$ denotes the set of functions from $A$ to $B$. If $f \in{ }^{A} B$ and $X \subseteq A$ then $f \uparrow X$ denotes the restriction of $f$ to $X$. We denote by Do $f$ and $\operatorname{Rg} f$ the domain and range of a given function $f$, respectively. We frequently identify $f$ with the sequence $\left\langle f_{x}: x \in\right.$ Do $\left.f\right\rangle$. We write $f x$ or $f_{x}$ or $f(x)$ to denote the value of $f$ at $x$. We define composition so that the right hand function acts first, thus for given functions $f, g, f \circ g(x)=f(g(x))$ whenever the left hand side is defined, i.e.c when $g(x) \in \operatorname{Do} f$.

Let $X$ be a set. Then $f(X)$ denotes the image of $X$ under $f$, i.e. $f(X)=$ $\{f(x): x \in X\} .|X|$ denotes the cardinality of $X$ and $\operatorname{Id}_{X}$, or simply $\mathrm{Id}$ when $X$ is clear from context, denotes the identity function on $X$. A set $X$ is countable if $|X| \leq \omega$. Let $Y$ be a set. Then $X \subseteq_{\omega} Y$ denotes that $X$ is a finite subset of $Y$. For a given class $K$ of algebras, $\mathbf{I} K$ denotes the class obtained by taking all isomorphic copies of algebras in $K$. Finally, given algebras $\mathcal{A}$ and $\mathcal{B}$ having the same signature, we let $\operatorname{Ism}(\mathcal{A}, \mathcal{B})$ denote the set of all isomorphisms of $\mathcal{A}$ into $\mathcal{B}$.

1.1. Construction of the algebras. $R$ denotes the set of real numbers. Let $U=R \times 3$. Note that $U$ is simply 3 disjoint copies of $R$. $\mathcal{C}$ denotes the full polyadic set algebra with unit ${ }^{2} U$, that is,

$$
\mathcal{C}=\left\langle\wp\left({ }^{2} U\right), \cap, \cup, \backslash, \emptyset,{ }^{2} U, c_{i}, d_{i j}, p_{i j}, s_{i}^{j}\right\rangle_{i, j<2} .
$$

Let $u=\left\langle u_{0}, u_{1}\right\rangle \in{ }^{2} 3$. For $r \in R$, we define $p(u, r)$ to be the following binary relation on $U$ :

$$
p(u, r)=\left\{\left\langle\left\langle a_{0}, u_{0}\right\rangle,\left\langle a_{1}, u_{1}\right\rangle\right\rangle \in{ }^{2} U: a_{0}=a_{1}+r\right\}
$$

and we let

$$
P(u)=\{p(u, r): r \in R\} .
$$

Note that $P(u)$ is an uncountable set of binary relations on $U$. Let $N$ be a fixed countably infinite subset of $R\left(^{4}\right)$. That is, $N \subseteq R$ and $|N|=\omega$. We set

$$
P_{\omega}(u)=\{p(u, r): r \in N\}
$$

$\left({ }^{4}\right)$ We point out that countability of $N$ is not essential. In fact any infinite subset $N$ of $R$ with $|N|<|R|$ will do just as well. 
The subscript $\omega$ indicates that $P_{\omega}(u)$ has countably many binary relations. Finally we let

$$
S=\left\{u \in{ }^{2} 3: u_{0} \leq u_{1}\right\}
$$

For an algebra $\mathcal{A}$ and $X \subseteq A, \operatorname{Sg}^{\mathcal{A}} X$ or simply $\operatorname{Sg} X$ denotes the subalgebra of $\mathcal{A}$ generated by $X$.

Now we define $\mathcal{A} \in Q E A s_{2}$ and $\mathcal{B} \subseteq \mathcal{A}$ as follows:

$$
\mathcal{A}=\operatorname{Sg}^{\mathcal{C}}(\bigcup\{P(u): u \in S\})
$$

and

$$
\mathcal{B}=\operatorname{Sg}^{\mathcal{A}}\left(\bigcup\{P(u): u \in S \backslash\{\langle 0,2\rangle\}\} \cup P_{\omega}(\langle 0,2\rangle)\right) .
$$

Plan of the proof. We will show that:

(1) $\mathcal{A} \in \mathbf{N} r_{2} W Q E A s_{\beta}$ for all $\beta>2$.

(2) $\mathcal{B}$ is an elementary subalgebra of $\mathcal{A}$.

(3) $\operatorname{Rd}_{S C} \mathcal{B} \notin \mathbf{I N} r_{2} S C_{\beta}$ for all $\beta>2$.

From (1)-(3), Theorem 1 will immediately follow by passing to reducts. In more detail, let $K \in\{S C, Q A, C A, Q E A\}$ and let $\beta>2$. Given $\mathcal{D} \in$ $Q E A_{\alpha}, \alpha$ an ordinal, let $\operatorname{Rd}_{K} \mathcal{D} \in K_{\alpha}$ denote the $K_{\alpha}$ reduct of $\mathcal{D}$. Then it follows from (1) that $\operatorname{Rd}_{K} \mathcal{A} \in N r_{2} W K s_{\beta}$, from (3) that $\operatorname{Rd}_{K} \mathcal{B} \notin \mathbf{I} N r_{2} K_{\beta}$ and from (2) that $\operatorname{Rd}_{K} \mathcal{B}$ is an elementary subalgebra of $\operatorname{Rd}_{K} \mathcal{A}$ (in the first order language of $K_{2}$ ).

1.2. $\mathcal{A}$ is a neat reduct. In this section we show that $\mathcal{A} \in \mathbf{N} r_{2} W Q E A s_{\beta}$ for all $\beta>2$. This will be done in two steps. First we show that $\mathcal{A} \in$ $\bigcap_{2 \leq k<\omega} \mathbf{N} r_{2} Q E A s_{k}$, then we show that $\mathcal{A} \in \mathbf{N} r_{2} W Q E A s_{\beta}$ for infinite $\beta$ using a limiting construction. Throughout, unless otherwise specified, $n=$ $\{0, \ldots, n-1\}$ denotes a finite ordinal $>1$.

Notation. $\mathbf{R}=\langle R,+,-, r\rangle_{r \in R}$ denotes the group of real numbers expanded with constants. $L$ denotes the first order language of $\mathbf{R}$. For $\phi$ an $L$-formula, we let $\operatorname{var}(\phi)$ denote the set of variables occurring in $\phi$, and we let $\operatorname{fr}(\phi)$ denote the set of variables occurring free in $\phi$.

Let $u=\left\langle u_{0}, \ldots, u_{n-1}\right\rangle \in{ }^{n} 3$. For $\phi$ an $L$-formula with $\operatorname{fr}(\phi) \subseteq\left\{x_{0}, \ldots\right.$ $\left.\ldots, x_{n-1}\right\}$, we let $E(u, \phi)$ denote the following $n$-ary relation on $U$ :

$$
E(u, \phi)=\left\{\left\langle\left\langle a_{0}, u_{0}\right\rangle, \ldots,\left\langle a_{n-1}, u_{n-1}\right\rangle\right\rangle \in{ }^{n} U: \mathbf{R} \models \phi\left[a_{0}, \ldots, a_{n-1}\right]\right\} .
$$

We let

$$
\begin{aligned}
& P(n)=\left\{E\left(u, x_{i}=x_{j}+r\right): u \in{ }^{n} 3, r \in R \text { and } i, j \in n, i<j\right\}, \\
& \mathcal{A}(n)=\operatorname{Sg}^{\mathcal{C}(n)}(P(n)),
\end{aligned}
$$

where $\mathcal{C}(n)$ is the full $Q E A s_{n}$ with unit ${ }^{n} U$.

It is easy to see that $\mathcal{A}=\mathcal{A}(2)$. 
We will show that for $1<l<m<\omega, \mathcal{A}(l) \cong \mathcal{N} r_{l} \mathcal{A}(m)$. For that we need some more definitions:

$$
F(n)=\left\{x_{i}=x_{j}+r, x_{i} \neq x_{j}+r: i, j \in n \text { and } r \in R\right\} .
$$

Forming finite conjunctions of formulas in $F(n)$, we let

$$
F(n)^{*}=\{\bigwedge J: J \subseteq \omega F(n)\} .
$$

Forming finite disjunctions of formulas in $F(n)^{*}$, we let

$$
F(n)^{* *}=\left\{\bigvee J: J \subseteq \omega F(n)^{*}\right\} .
$$

Now set

$$
G(n)=\left\{E(u, \phi): u \in^{n} 3 \text { and } \phi \in F(n)^{* *}\right\}
$$

and finally

$$
G(n)^{*}=\{\bigcup J: J \subseteq \omega G(n)\} .
$$

FACT 1.2.1. Let $n>1$. Then $A(n)=G(n)^{*}$.

Proof. First we prove that $A(n) \subseteq G(n)^{*}$. Since $P(n) \subseteq G(n)^{*}$ and $\mathcal{A}(n)$ is generated by $P(n)$, it suffices to show that $G(n)^{*}$ is the universe of a $Q E A_{n}$, i.e. is closed under the polyadic set operations.

(1) $G(n)^{*}$ is closed under the boolean operations. Indeed, let $u, v \in{ }^{n} 3$ and $\phi_{1}, \phi_{2} \in F(n)^{* *}$. Then

$$
E\left(u, \phi_{1}\right) \cap E\left(v, \phi_{2}\right)=E\left(u, \phi_{1} \wedge \phi_{2}\right) \quad \text { if } u=v
$$

and is zero otherwise, while

$$
{ }^{n} U \backslash E\left(u, \phi_{1}\right)=\bigcup\left\{E(v, \top): v \in{ }^{n} 3 \backslash\{u\}\right\} \cup E\left(u, \neg \phi_{1}\right) .
$$

From the definition of $G(n)^{*}$, and by noting that for $\phi_{1}$ and $\phi_{2}$ in $F(n)^{* *}$, there exist $\eta$ and $\theta$ in $F(n)^{* *}$ such that $\mathbf{R} \models \phi_{1} \wedge \phi_{2} \leftrightarrow \eta$ and $\mathbf{R} \models \neg \phi_{1} \leftrightarrow \theta$, we get the desired conclusion.

(2) $G(n)^{*}$ contains the diagonal elements. Let $i<j<n$. Then

$$
d_{i j}=\bigcup\left\{E\left(u, x_{i}=x_{j}\right): u \in{ }^{n} 3, u_{i}=u_{j}\right\} .
$$

and so by the definition of $F(n)$ we get $d_{i j} \in G(n)^{*}$.

(3) $G(n)^{*}$ is closed under cylindrifications. First a piece of useful notation. For $u, v \in{ }^{n} 3$ and $i<n$ we write $u \equiv_{i} v$ iff $u$ and $v$ agree off $i$, i.e. $u(j)=v(j)$ for all $j \neq i$. Now let $g \in G(n)^{*}$ and $i<n$. Since the $c_{i}$ 's $(\exists)$ distribute(s) over joins $(\vee)$ we can and will assume that $g=E(u, \phi)$ with $u \in{ }^{n} 3$ and $\phi \in F(n)^{* *}$. Computing we get

$$
c_{i} g=c_{i} E(u, \phi)=\bigcup\left\{E\left(v, \exists x_{i} \phi\right): v \equiv_{i} u\right\} .
$$

Now we make use of the following consequence of the fact that $\mathbf{R}$ is in a sense "saturated", which we abbreviate by $(*)$ :

(*) Given $\phi \in F(n)^{* *}$ and $i<n$, there exists $\psi \in F(n)^{* *}$ such that

$$
x_{i} \notin \operatorname{var}(\psi) \quad \text { and } \quad \mathbf{R}=\exists x_{i} \phi \leftrightarrow \psi \text {. }
$$


Note that $(*)$ is a form of elimination of quantifiers. Now applying $(*)$ we see that $c_{i} g \in G(n)^{*}$.

(4) $G(n)^{*}$ is closed under substitutions. Since $s_{j}^{i}(x)=c_{i}\left(x \cap d_{i j}\right)$ for $i \neq j$ and $s_{i}^{i}(x)=x$, so that the $s_{j}^{i}$ 's are term definable, it suffices to check only those substitutions of the form $p_{i j}$. So let $\phi \in F(n)^{* *}, u \in{ }^{n} 3$ and $i, j<n$ be distinct. Abusing notation slightly, we let $p_{i j} \phi$ stand for the formula obtained from $\phi$ by interchanging the free occurrences of $x_{i}$ and $x_{j}$, and we let $p_{i j} u=u \circ[i, j]$. Since $p_{i j}$ is a boolean endomorphism, it suffices to show that for all $u \in{ }^{n} 3$ and all $\phi \in F(n)$ we have $p_{i j} E(u, \phi) \in G(n)^{* *}$. But this follows from the facts that

$$
p_{i j} E(u, \phi)=E\left(p_{i j} u, p_{i j} \phi\right)
$$

and that $p_{i j} \phi \in F(n)$ for $\phi \in F(n)$.

Next we show that $G(n)^{*} \subseteq A(n)$. It clearly suffices to show that for all $u \in{ }^{n} 3$ and $\phi \in F(n)$, we have $E(u, \phi) \in A(n)$. Let $u$ and $\phi$ be as indicated. Suppose that $\phi$ is $x_{i}=x_{j}+r$ for some $i, j \in n$ and some $r \in R$. If $i<j$, then $E(u, \phi) \in P(n)$. If $i>j$ then $E(u, \phi)=E\left(u, x_{j}=x_{i}+(-r)\right)$, which also belongs to $P(n)$. Thus we are left with the case $i=j$. We have two subcases.

Case a. $r \neq 0$ and so $E(u, \phi)=0 \in A(n)$.

Case b. $r=0$, then $E(u, \phi)=E(u, \top)$. In order to show that the latter belongs to $A(n)$, we let $k \in n \backslash\{i\}$. This is possible because $n>1$. Then a straightforward computation gives

$$
c_{i} E\left(u, x_{i}=x_{k}\right) \cap c_{k} E\left(u, x_{i}=x_{k}\right)=E(u, \top),
$$

thus the latter is in $A(n)$. Finally by noting that $E(u, \neg \phi)=E(u, \top) \backslash$ $E(u, \phi)$ we infer (using the above reasoning) that $E\left(u, x_{i} \neq x_{j}+r\right)$ is also in $A(n)$, for all $u \in{ }^{n} 3$ and $i, j \in n$.

Having shown that $G(n)^{*}=A(n)$, and in particular that $G(n)^{*}$ is closed under the $Q E A_{n}$ operations, we let $\mathcal{G}(n)^{*}$ denote the $Q E A s_{n}$ with universe $G(n)^{*}$.

For further use we shall need

Definition. For $m \geq 1$, let $\mathcal{C}(m)$ denote the full $Q E A s_{m}$ with unit ${ }^{m} U$, i.e. the universe of $\mathcal{C}(m)$ is $\wp\left({ }^{m} U\right)$. Now let $1 \leq l<k$. Let $i(l, k)$ be the following (neat embedding) function:

$$
i(l, k): C(l) \rightarrow C(k), \quad a \mapsto\left\{t \in{ }^{k} U: t\lceil l \in a\} .\right.
$$

FACT 1.2.2. Let $1<l<k<\omega$. Then $i(l, k) \mathcal{G}(l)^{*}=\mathcal{N} r_{l} \mathcal{G}(k)^{*}$. In particular, $\mathcal{A} \in \bigcap_{2 \leq k<\omega} \mathbf{N} r_{2} Q E A s_{k}$. 
Proof. Let $1 \leq l<k$. Assume that $k$, hence $l$, is finite. Then it is easy to check that

$$
i(l, k)\left\lceil G(l)^{*} \in \operatorname{Ism}\left(\mathcal{G}(l)^{*}, \mathcal{N} r_{l} \mathcal{C}(k)\right) .\right.
$$

We claim that, in fact, we have $i(l, k) G(l)^{*} \subseteq G(k)^{*}$. To see this, let $u \in{ }^{l} 3$ and $\phi \in F(l)$. Then

$$
\begin{aligned}
i(l, k) E(u, \phi) & =\left\{s \in{ }^{k} U: s\lceil l \in E(u, \phi)\}\right. \\
& =\bigcup\left\{E(v, \phi): v \in{ }^{k} 3 \text { and } v\lceil l \subseteq u\} .\right.
\end{aligned}
$$

The latter is in $G(k)^{*}$ because $F(l) \subseteq F(k), \phi \in F(l)$ and $k<\omega$. Since $\mathcal{G}(k)^{*}$ is a $Q E A s_{k}$, we find that $i(l, k) E(u, \phi) \in G(k)^{*}$ for any $u \in{ }^{l} 3$ and $\phi \in F(l)^{* *}$. As $N r_{l} G(k)^{*}=G(k)^{*} \cap N r_{l} C(k)$, we deduce that

$$
i(l, k)\left\lceil G(l)^{*} \in \operatorname{Ism}\left(\mathcal{G}(l)^{*}, \mathcal{N} r_{l} \mathcal{G}(k)^{*}\right) .\right.
$$

Next we show that $i(l, k)$ is actually onto $\mathcal{N} r_{l} \mathcal{G}(k)^{*}$, i.e. $i(l, k) G(l)^{*}=$ $N r_{l} G(k)^{*}$. This will be proved by using the fact that the structure $\mathbf{R}$ admits elimination of quantifiers as expressed in $(*)$ above. In more detail, since the $c_{i}$ 's are additive, it suffices to show that for all $g \in G(k)$, there exists $a \in G(l)^{*}$ such that

$$
i(l, k) a=c_{l} c_{l+1} \ldots c_{k-1} g .
$$

So let $g=E(u, \phi)$ be as specified with $u \in{ }^{k} 3$ and $\phi \in F(k)^{* *}$. Suppose further that $\phi=\phi_{0} \vee \phi_{1} \vee \ldots \vee \phi_{m}$ with $\phi_{j} \in F(k)^{*}$ for $0 \leq j \leq m$. For brevity set $v=u \uparrow l$. Note that $v \in{ }^{l} 3$. Then an easy computation gives, for every $j \leq m$,

$$
c_{l} c_{l+1} \ldots c_{k-1} E\left(u, \phi_{j}\right)=i(l, k) E\left(v, \exists x_{l} \exists x_{l+1} \ldots \exists x_{k-1} \phi_{j}\right) .
$$

Now $(*)$ guarantees the existence of $\psi_{j} \in F(l)^{* *}$ for every $j \leq m$ such that

$$
\mathbf{R} \models \exists x_{l} \exists x_{l+1} \ldots \exists x_{k-1} \phi_{j} \leftrightarrow \psi_{j} .
$$

Now computing we get

$$
\begin{aligned}
& c_{l} c_{l+1} \ldots c_{k-1} E(u, \phi)=c_{l} c_{l+1} \ldots c_{l} \ldots c_{k-1} E\left(u, \phi_{0} \vee \ldots \vee \phi_{m}\right) \\
& =c_{l} c_{l+1} \ldots c_{k-1} E\left(u, \phi_{0}\right) \cup \ldots \cup c_{l} c_{l+1} \ldots c_{k-1} E\left(u, \phi_{m}\right) \\
& =i(l, k) E\left(v, \psi_{0}\right) \cup \ldots \cup i(l, k) E\left(v, \psi_{m}\right)=i(l, k) E\left(v, \psi_{0} \vee \ldots \vee \psi_{m}\right) .
\end{aligned}
$$

Since by definition $\psi_{0} \vee \ldots \vee \psi_{m} \in F(l)^{* *}$, we are done.

FACT 1.2.3. Let $2 \leq n<\omega$ and $\beta$ be infinite. Then $\mathcal{G}(n)^{*} \in \mathbf{N} r_{n} Q E A_{\beta}$. In particular, $\mathcal{A} \in \mathbf{N} r_{2} Q E A_{\alpha}$ for every $\alpha>2$.

Proof. Fix $\beta \geq \omega$. Let

$$
\mathcal{G}(\beta)=\operatorname{Sg}^{\mathcal{C}(\beta)}\left(\bigcup\left\{i(k, \beta) G(k)^{*}: n \leq k<\omega\right\}\right) .
$$

Here, as in Fact 1.2.2, $\mathcal{C}(\beta)$ denotes the full $C s_{\beta}$ with unit ${ }^{\beta} U$; and for $a \in G(k)^{*}$ recall that $i(k, \beta) a=\left\{s \in{ }^{\beta} U: s \uparrow k \in a\right\}$. Let $a \in G(\beta)$. We will 
show that $a \in N r_{n} G(\beta)$ iff $a \in i(n, \beta) G(n)^{*}$, from which it readily follows that $i(n, \beta) G(n)^{*}=N r_{n} G(\beta)$, and so $\mathcal{G}(n)^{*} \cong \mathcal{N} r_{n} \mathcal{G}(\beta)$. First note that $\bigcup\left\{i(k, \beta) G(k)^{*}: n \leq k<\omega\right\}$ is a subuniverse of $G(\beta)$. This is straightforward. Thus

$$
G(\beta)=\bigcup\left\{i(k, \beta) G(k)^{*}: n \leq k<\omega\right\} .
$$

It then follows that $a=i(l, \beta) g$ for some $n \leq l<\omega$ and $g \in G(l)^{*}$. Now suppose that $a \in N r_{n} G(\beta)$. Then $g=c_{j} g$ for all $n \leq j<\omega$ and so $g \in$ $N r_{n} G(l)^{*}$. By Fact 1.2.2 we have $N r_{n} G(l)^{*}=i(n, l) G(n)^{*}$; thus $g=i(n, l) g^{\prime}$ say, for some $g^{\prime} \in G(n)^{*}$. But

$$
a=i(l, \beta) g=i(l, \beta) \circ i(n, l) g^{\prime}=i(n, \beta) g^{\prime} .
$$

This shows that $a \in i(n, \beta) G(n)^{*}$. Conversely if $a \in i(n, \beta) G(n)^{*}$, then it is easy to see that $a \in N r_{n} G(\beta)$. We have shown that $i(n, \beta) G(n)^{*}=$ $N r_{n} G(\beta)$. Since $\mathcal{A} \cong \mathcal{G}(2)^{* *}$, and $\mathcal{G}(\beta) \in Q E A_{\beta}$, we conclude that $\mathcal{A} \in$ $\mathrm{N} r_{2} Q E A_{\beta}$ for all $\beta>2$.

In Facts 1.2.2-3, we have proved that $\mathcal{A} \in \bigcap_{2 \leq k<\omega} \mathbf{N} r_{2} Q E A s_{k}$ and that $\mathcal{A} \in \mathbf{N} r_{2} Q E A_{\beta}$ for infinite $\beta$, as well. In Fact 1.2.4 below we show that we can replace the class $Q E A_{\beta}$ by the smaller classes $W Q E A s_{\beta}$ and $Q E A s_{\beta}$ for infinite $\beta$. For this we need a definition.

Definition. Let $\beta$ be an ordinal. We recall from [16, Def. 3.1.1(viii)] that $\mathcal{A} \in K s_{\beta}$ with unit ${ }^{\beta} U$ is regular if for all $x \in A$ and all $f, g \in{ }^{\beta} U$, whenever $f\left\lceil\Delta x \subseteq g\right.$ and $f \in X$ then $g \in X . \mathcal{A} \in K_{\beta}$ is locally finite if $\Delta x$ is finite for every element in $A$.

Notation. Let $\beta$ be an infinite ordinal. Then $K s_{\beta}^{\text {reg }}$ and $\operatorname{Lf} K_{\beta}$ stand for the classes of regular $K$ set algebras, and locally finite $K$ algebras of dimension $\beta$, respectively.

It is known (cf. $[16,3.1 .70])\left({ }^{5}\right)$ that for infinite $\beta$ we have

$$
K s_{\beta}^{\text {reg }} \cap \operatorname{Lf} K_{\beta} \subseteq I W K s_{\beta} .
$$

FACT 1.2.4. $\mathcal{G}(n)^{*} \in \mathbf{N} r_{n} W Q E A s_{\beta}$ for infinite $\beta$. In particular, $\mathcal{A} \in \mathbf{N} r_{2} W Q E A s_{\beta}$ for infinite $\beta$.

Proof. Fix $\beta \geq \omega$. Let $\mathcal{F}(\beta)=\operatorname{Sg}^{\mathcal{G}(\beta)} i(n, \beta)\left(\mathcal{G}(n)^{*}\right)$, where $\mathcal{G}(\beta)$, as usual, denotes the full quasipolyadic set algebra with unit ${ }^{\beta} U$. Then, as is easily checked, we have $\mathcal{F}(\beta) \in Q E A s_{\beta}^{\text {reg }} \cap \operatorname{Lf} Q E A_{\beta}$, and $\mathcal{G}(n)^{*} \cong \mathcal{N} r_{n} \mathcal{F}(\beta)$. Since $I Q E A s_{\beta}^{\mathrm{reg}} \cap \operatorname{Lf} Q E A_{\beta} \subseteq I W Q E A s_{\beta}$, we are done.

Remarks. (1) The class Lf $K_{\beta} \cap K s_{\beta}^{\text {reg }}$ is the algebraic counterpart of first order models. For more on such connections the reader is referred to [24],

$\left({ }^{5}\right)$ We should point out that Theorem 3.1.70 in [16] is formulated only for cylindric algebras. However the proof easily adapts to $S C$ 's, $Q A$ 's and $Q E A$ 's, as shown for example in [29] or [3]. 
[16, Sec. 4.3], [3] and [29, Chapter 4]. In fact, one can define an isomorphism $h:$ Models $\rightarrow \operatorname{Lf} K_{\omega} \cap K s_{\omega}^{\text {reg }}$ definable in $Z F C$ by an absolute formula without parameters (cf. [23, p. 406]).

(2) Following the referee, we give a simpler description of the algebra $\mathcal{A}$. Consider a first order language $L$ consisting of binary relation symbols $\delta_{u}^{r}$ for all $u \in{ }^{2} 3$ and all real numbers $r$. Let $M$ be the $L$-structure with domain consisting of three disjoint copies $R_{0}, R_{1}, R_{2}$ of the real numbers, and with

$$
M \models \delta_{u}^{r}(a, b) \quad \text { iff } \quad a \in R_{u_{0}}, b \in R_{u_{1}} \text {, and } b-a=r .
$$

Now $\mathcal{A}$ is isomorphic to the first order definable subsets of ${ }^{2} M$. In more detail, for $\beta>2$, any $v \in{ }^{\beta} M$ can be regarded as an assignment of variables $x_{i}(i<\beta)$ to $M$. Let $\mathcal{D}$ be the set of all subsets of ${ }^{\beta} M$ of the form $\{v \in$ $\left.{ }^{\beta} M: M=\phi[v]\right\}$ where $\phi$ is a first order $L$-formula written with variables $x_{i}, i<\beta$, and the operations are defined as for set algebras. Here we are adopting the usual notion: $M=\phi[v]$ if $v$ satisfies $\phi$ in $M$. Then, of course, $\mathcal{D} \in Q E A s_{\beta}$. In fact, $\mathcal{D} \in \operatorname{Lf} Q E A_{\beta} \cap Q E A s_{\beta}^{\text {reg }}$. On the other hand, $\mathcal{A}$ is isomorphic to the set algebra consisting of all subsets of ${ }^{2} M$ of the form $\left\{v \in{ }^{2} M: M \models \phi[v]\right\}$ where $\phi$ is an $L$-formula written with two variables $x_{0}, x_{1}$, and with operations defined by restricting those of $\mathcal{D}$ to the first two dimensions. By the proof of Fact 1.2.1, $M$ has quantifier elimination, so $\mathcal{A} \cong \mathcal{N} r_{2} \mathcal{D}$. This also proves Fact 1.2.4.

Henceforth we only deal with the 2-dimensional polyadic set algebras $\mathcal{A}$ and $\mathcal{B}$.

1.3. $\mathcal{B}$ is an elementary subalgebra of $\mathcal{A}$. In this section we show that the $Q E A s_{2} \mathcal{A}$ admits a particularly rich set of automorphisms, a property we use to show that $\mathcal{B}$ is elementarily equivalent to $\mathcal{A}$.

Notation. For $u=\left\langle u_{0}, u_{1}\right\rangle \in{ }^{2} 3$ we let $1_{u_{0} u_{1}}$, and sometimes simply $1_{u}$, denote $E(u, \top)$. For $a \in A$ and $X \subseteq A$, we let $\mathrm{Rl}_{a} X$ denote the relativization of $X$ to $a$, that is, $\mathrm{Rl}_{a} X=\{x \in X: x \leq a\}$.

FACT 1.3.1. Let $u \in{ }^{2} 3$. For brevity, set $V=E(u, \top)$. Then:

(i) $\mathrm{Rl}_{V} A=\left\{E(u, \phi): \phi \in F(u)^{* *}\right\}$.

(ii) $\mathrm{Rl}_{V} A$ is an atomic boolean algebra and the set of its atoms is equal to $P(u)=\{p(u, r): r \in R\}$. In particular, $\mathrm{Rl}_{V} A$ (as a boolean algebra) is generated by its atoms.

(iii) For all non-zero $a \in \mathrm{Rl}_{V} A$ and for all $i<2$ we have $c_{i} a=c_{i} V$.

(iv) For all $a \in A$ and for all $i<2$ we have $c_{i} a \cap V \in\{0, V\}$.

Proof. (i) follows from the fact that $\mathcal{A}=\mathcal{G}(2)$. (ii) follows from the fact that for $u \in{ }^{2} 3$ and $r_{1}, r_{2}$ distinct elements of $R$ we have

$$
p\left(u, r_{1}\right) \cap p\left(u, r_{2}\right)=0 .
$$

(iv) follows from (iii). Now we prove (iii). 
Let $a \in \mathrm{Rl}_{V} A$. Then $a \leq V$. By (i), $a=E(u, \phi)$ for some $\phi \in F(2)^{* *}$. Now suppose that $0 \neq a$ and $i<2$. Then by (ii) there exists an atom in $\mathrm{Rl}_{V} A$ below $a$, i.e. an $r \in R$ such that $E\left(u, x_{0}=x_{1}+r\right)=p(u, r) \leq a$. Now we have

$$
\begin{aligned}
c_{i} a & \leq c_{i} V=\bigcup\left\{E(v, \top): v \in{ }^{2} 3, v \equiv_{i} u\right\} \\
& =c_{i} E\left(u, x_{0}=x_{1}+r\right)=c_{i}(p(u, r)) \leq c_{i} a .
\end{aligned}
$$

We have proved (iii).

REMARK. Notice that the set $\left\{1_{u}: u \in{ }^{2} 3\right\}$ forms a partition of the unit $1=\bigcup\left\{1_{u}: u \in{ }^{2} 3\right\}$ of the algebra $\mathcal{A}$ so that the boolean structure of $\mathcal{A}$ is rather simple. It is isomorphic to the product of $\mathrm{Rl}_{1_{u}} A$ indexed by ${ }^{2} 3$, the isomorphism being

$$
h(a)=\left\langle a \cap 1_{u}: u \in{ }^{2} 3\right\rangle \quad \text { for } a \in A .
$$

$\mathrm{Rl}_{1_{u}} A$ has an even simpler boolean structure. It is isomorphic to the boolean algebra (with universe) $\wp(R)$ generated by the singletons, which is the same as the finite co-finite boolean algebra on $R$, i.e. the algebra with universe

$$
\{X \subseteq R: X \text { or } R \backslash X \text { is finite }\} .
$$

Note too that $P(u)$ constitutes further an uncountable partition of $1_{u}$ that is a splitting of $1_{u}$ in the sense of Andréka [2]. This means that every element in $P(u)$ is cylindrically equivalent to $1_{u}$, i.e. $c_{i} x=c_{i} 1_{u}$ for every $i<2$ and every $x \in P(u)$. So in a sense (at the least in the above one) the elements in $P(u)$ are "big". Being atoms in $\mathrm{Rl}_{1_{u}} A$ (and even more, as is easily checked, in $\mathcal{A}$ ), they are also "small". Andréka [2] refers to such elements as big atoms. It is precisely the "duality" in the nature of such elements (the big atoms) that will enable us, in Fact 1.3.2 below, to extend given permutations on the set $P=\left\{p(u, r): u \in{ }^{2} 3, u_{0} \leq u_{1}, r \in R\right\}$ to automorphisms on $\mathcal{A}$. $\mathcal{A}$ in this respect resembles the free structure on $P$.

FACT 1.3.2. Let $P=\left\{p(u, r): u \in{ }^{2} 3, u_{0} \leq u_{1}, r \in R\right\}$. Let $f$ be $a$ permutation of $P(\langle 0,2\rangle)$. Then there exists an automorphism $h$ of $\mathcal{A}$ such that $f \subseteq h$ and $h \uparrow(P \backslash[P(\langle 0,2\rangle) \cup P(\langle 2,0\rangle)])=\mathrm{Id}$.

Proof. Let $f$ be a given permutation of $P(\langle 0,2\rangle)$. Define

$$
f^{*}=p_{01} \circ f \circ p_{01} \text {. }
$$

Then, as is easily checked, $f^{*}$ is a permutation of $P(\langle 2,0\rangle)$. By Fact 1.3.1, $\mathrm{Rl}_{1_{02}} A$ is generated as a boolean algebra by the set $P(\langle 0,2\rangle)$ of its atoms. It follows that there exists an automorphism $h_{1}$ of $\mathrm{BlRl}_{1_{02}} A$ such that $f \subseteq h_{1}$. Similarly there exists an automorphism $h_{2}$ of $\mathrm{BlRl}_{1_{20}} A$ such that $f^{*} \subseteq h_{2}$. For $x \in A$ let

$$
h(x)=h_{1}\left(x \cap 1_{02}\right) \cup h_{2}\left(x \cap 1_{20}\right) \cup \bigcup\left\{x \cap 1_{u}: u \in{ }^{2} 3 \text { and } \operatorname{Rg} u \neq\{0,2\}\right\} .
$$


We show that $h$ is the desired automorphism. Clearly $h$ is a boolean automorphism that extends $f$ and is the identity on $P \backslash[P(\langle 0,2\rangle) \cup P(\langle 2,0\rangle)]$. So all we have to check is that $h$ also preserves the extra non-boolean operations.

(1) $h$ preserves the diagonal element. Indeed,

$h\left(d_{01}\right)=h_{1}\left(d_{01} \cap 1_{02}\right) \cup h_{2}\left(d_{01} \cap 1_{20}\right) \cup \bigcup\left\{d_{01} \cap 1_{u}: u \in{ }^{2} 3\right.$ and $\left.\operatorname{Rg} u \neq\{0,2\}\right\}$.

Since $d_{01} \cap 1_{02}=d_{01} \cap 1_{20}=0$, and $h_{1}, h_{2}$ are automorphisms of $\mathrm{Rl}_{1_{02}} A$ and $\mathrm{Rl}_{1_{20}} A$, respectively, so that $h_{1}\left(d_{01} \cap 1_{02}\right)=h_{2}\left(d_{01} \cap 1_{20}\right)=0$, we have

$$
h\left(d_{01}\right)=\bigcup\left\{d_{01} \cap 1_{u}: u \in{ }^{2} 3 \text { and } \operatorname{Rg} u \neq\{0,2\}\right\}=d_{01} .
$$

(2) $h$ preserves $p_{01}$. Let $Y=\left\{p(u, r): u \in{ }^{2} 3, r \in R\right\}$. Let $X=\left\{c_{i} a\right.$ : $a \in A, i<2\} \cup\left\{d_{01}\right\}$, and let $\mathcal{D}$ be the boolean algebra generated by $X \cup Y$. We claim that $D$ is a subuniverse of $\mathcal{A}$. Since $D$ contains $d_{01}$ and is closed under the boolean set operations, it suffices to show that $D$ is closed under $c_{0}, c_{1}$ and $p_{01}$. So let $a \in D$ and $i<2$. As $D \subseteq A$ we have $a \in A$ and so, by definition, $c_{i} a \in D$. Since $p_{01}$ is a boolean endomorphism, it suffices to check that for $x \in Y \cup X$ we have $p_{01} x \in D$. But this is true because $x \in Y$ iff $p_{01} x \in Y, x \in X$ iff $x=0$ or there is a subset $J$ of ${ }^{2} 3$ such that $x=\left\{1_{u}: u \in J\right\}$ and $p_{01} 1_{u_{0} u_{1}}=1_{u_{1} u_{0}}$. Thus we have shown that $D$ is a subuniverse of $\mathcal{A}$. Since $Y \subseteq D, Y$ generates $\mathcal{A}$, and $D \subseteq A$, we get $D=A$. Now let $X^{\prime}=\left\{1_{u}: u \in{ }^{2} 3\right\} \cup\left\{d_{01}\right\}$ and let $\mathcal{D}^{\prime}$ be the boolean algebra generated by $X^{\prime} \cup Y$. Then $D=D^{\prime}$ because every non-zero element of $X$ is a finite union of elements in $X^{\prime}$. Thus $D^{\prime}=A$. Since $p_{01}$ is a boolean endomorphism, to prove that $h$ preserves $p_{01}$, it is enough to show that $h\left(p_{01} x\right)=p_{01} h(x)$ for all $x \in Y \cup X^{\prime}$. To this end, let $x \in Y \cup X^{\prime}$. If $u \in{ }^{2} 3$, $\operatorname{Rg} u \neq\{0,2\}$ and $x \in\left\{p(u, r), 1_{u}\right\}$, then

$$
p_{01} x \cap 1_{v}=0 \quad \text { for } v \in\{\langle 0,2\rangle,\langle 2,0\rangle\}
$$

thus

$$
\begin{aligned}
h\left(p_{01} x\right) & =\bigcup\left\{p_{01} x \cap 1_{u}: u \in{ }^{2} 3 \text { and } \operatorname{Rg} u \neq\{0,2\}\right\} \\
& =p_{01} x=p_{01} h(x) \quad(\text { since } h(x)=x) .
\end{aligned}
$$

Else

$$
x=p(u, r) \quad \text { or } \quad x=1_{u} \quad \text { with } \quad \operatorname{Rg} u=\{0,2\} .
$$

Suppose that $u=\langle 0,2\rangle$ and that $x=p(\langle 0,2\rangle, r)$. (The subcases $x=1_{02}$ or $x=1_{20}$ or $x=p(\langle 2,0\rangle, r)$ can be treated analogously and are left to the reader.) By noting that $p_{01} x=p(\langle 2,0\rangle,-r)$ and computing we get

$$
\begin{aligned}
h\left(p_{01} x\right) & =h_{2}(p(\langle 2,0\rangle,-r))=f^{*}(p(\langle 2,0\rangle,-r))=p_{01} \circ f \circ p_{01}[p(\langle 2,0\rangle,-r)] \\
& =p_{01} \circ f \circ p_{01} \circ p_{01}(x)=p_{01} \circ f(x)=p_{01}(h(x)) .
\end{aligned}
$$


(3) Finally we check cylindrifications. Let $k<2$ and $x \in A$. Then computing we get

$$
\begin{aligned}
& c_{k} h(x) \\
& =c_{k}\left(h_{1}\left(x \cap 1_{02}\right) \cup h_{2}\left(x \cap 1_{20}\right) \cup \cup_{u \in{ }^{2} 3}\left\{x \cap 1_{u}: \operatorname{Rg} u \neq\{0,2\}\right\}\right) \\
& =c_{k} h_{1}\left(x \cap 1_{02}\right) \cup c_{k} h_{2}\left(x \cap 1_{20}\right) \cup \bigcup_{u \in{ }^{2} 3}\left\{c_{k}\left(x \cap 1_{u}\right): \operatorname{Rg} u \neq\{0,2\}\right\} .
\end{aligned}
$$

Since $h_{1}$ is an automorphism of $\mathrm{Rl}_{1_{02}} A$ and $x \cap 1_{02} \leq 1_{02}$, we get

$$
h_{1}\left(x \cap 1_{02}\right) \leq h_{1}\left(1_{02}\right)=1_{02} .
$$

Since $x \cap 1_{02}=0$ iff $h_{1}\left(x \cap 1_{02}\right)=0$, by Fact 1.3.1(iii) we have

$$
c_{k}\left(h_{1}\left(x \cap 1_{02}\right)\right)=c_{k}\left(x \cap 1_{02}\right) .
$$

By exactly the same reasoning it follows that

$$
c_{k}\left(h_{2}\left(x \cap 1_{20}\right)\right)=c_{k}\left(x \cap 1_{20}\right) .
$$

Substituting in $(*)$ we obtain

$$
\begin{aligned}
c_{k} h(x) & =c_{k}\left(x \cap 1_{02}\right) \cup c_{k}\left(x \cap 1_{20}\right) \cup \bigcup_{u \in{ }^{2} 3}\left\{c_{k}\left(x \cap 1_{u}\right): \operatorname{Rg} u \neq\{0,2\}\right\} \\
& =c_{k}\left(x \cap \bigcup\left\{1_{u}: u \in{ }^{2} 3\right\}\right)=c_{k} x .
\end{aligned}
$$

Now computing $h\left(c_{k} x\right)$ we get

$$
\begin{aligned}
& h\left(c_{k} x\right)= h_{1}\left(c_{k} x \cap 1_{02}\right) \cup h_{2}\left(c_{k} x \cap 1_{20}\right) \\
& \cup \bigcup_{u \in{ }^{2} 3}\left\{c_{k} x \cap 1_{u}: \operatorname{Rg} u \neq\{0,2\}\right\} .
\end{aligned}
$$

That $h_{1}\left(c_{k} x \cap 1_{02}\right)=c_{k} x \cap 1_{02}$ and $h_{2}\left(c_{k} x \cap 1_{20}\right)=c_{k} x \cap 1_{20}$ follows from Fact 1.3.1(iv) upon noting that $h_{1}$ and $h_{2}$ are boolean automorphisms; thus we have

$$
h\left(c_{k} x\right)=c_{k} x \cap \bigcup\left\{1_{u}: u \in{ }^{2} 3\right\}=c_{k} x .
$$

We have shown that $c_{k} h(x)=h_{c} k(x)=c_{k} x\left({ }^{6}\right)$, thus $h$ preserves cylindrifications.

FACT 1.3.3. $\mathcal{B}$ is an elementary subalgebra of $\mathcal{A}$.

Proof. We shall use the Tarski-Vaught criterion to show that $\mathcal{B}$ is an elementary subalgebra of $\mathcal{A}$. So let $\exists x_{n} \phi\left(x_{0}, \ldots, x_{n}\right)$ be any first order formula in the language of $Q E A_{2}$. Suppose that $b_{0}, \ldots, b_{n-1} \in B$, and that $\mathcal{A}=\phi\left(b_{0}, \ldots, b_{n-1}, a\right)$ for some $a \in A$. We want to find $b_{n} \in B$ such that $\mathcal{A}=\phi\left(b_{0}, \ldots, b_{n-1}, b_{n}\right)$. Since $\mathcal{A}$ is generated by $P=\left\{p(u, r): u \in{ }^{2} 3, u_{0} \leq\right.$ $\left.u_{1}, r \in R\right\}$, there exists a finite subset $H_{0}$ of $P$ such that $a \in \operatorname{Sg}^{\mathcal{A}} H_{0}$. Let

$$
P^{\prime}=\left\{p(u, r): u \in{ }^{2} 3, u_{0} \leq u_{1}, u \neq\langle 0,2\rangle, r \in R\right\} \cup P_{\omega}(\langle 0,2\rangle) .
$$

$\left({ }^{6}\right)$ We used the "bigness" condition to show that $h$ preserves cylindrifications. Loosely speaking, when a cylindric or polyadic algebra $\mathcal{A}$ is generated by a set of big atoms, then we can forget about cylindrifications when constructing automorphisms of $\mathcal{A}$. This method is also referred to as the method of eliminating cylindrifications. 
Since $b_{0}, \ldots, b_{n-1} \in B$, and $\mathcal{B}$ is generated by $P^{\prime}$, there exists a finite subset $H_{1}$ of $P^{\prime}$ such that $\left(H_{0} \cap B\right) \cup\left\{b_{0}, \ldots, b_{n-1}\right\} \subseteq \mathrm{Sg}^{\mathcal{B}} H_{1}$. Let $f$ be a permutation of $P(\langle 0,2\rangle)$ such that

$$
f \nmid P(\langle 0,2\rangle) \cap H_{1} \subseteq \mathrm{Id} \quad \text { and } \quad f\left(H_{0} \backslash H_{1}\right) \subseteq P_{\omega}(\langle 0,2\rangle) .
$$

Such a permutation exists, since $\left|H_{0}\right|<\omega,\left|H_{1}\right|<\omega$ and $\left|P_{\omega}(\langle 0,2\rangle)\right|=\omega$. By Fact 1.3.2, $f$ extends to an automorphism $h$ of $\mathcal{A}$ such that

$$
h \uparrow(P \backslash P(\langle 0,2\rangle)) \subseteq \mathrm{Id} .
$$

But

$$
\mathcal{A}=\phi\left(b_{0}, \ldots, b_{n-1}, a\right)
$$

and so

$$
\mathcal{A} \models \phi\left(h\left(b_{0}\right), \ldots, h\left(b_{n-1}\right), h(a)\right) .
$$

Since $h\left\lceil H_{1} \subseteq\right.$ Id and $\left\{b_{0}, \ldots, b_{n}\right\} \subseteq \operatorname{Sg}^{\mathcal{B}} H_{1}$, it follows that $h\left(b_{i}\right)=b_{i}$ for all $i<n$. Since $h\left(H_{0}\right) \subseteq B$ and $a \in \mathrm{Sg}^{\mathcal{A}} H_{0}$, we get $h(a) \in B$. This completes the proof.

REMARK. We have shown that first order logic $\left(L_{\omega, \omega}\right)$ cannot distinguish between $\mathcal{A}$ and $\mathcal{B}$. It can be proved that stronger logics like $L_{k, \omega}$ where $k$ is an infinite cardinal also cannot distinguish between $\mathcal{A}$ and $\mathcal{B}$. Recall that $L_{k, \omega}$ is obtained from $L_{\omega, \omega}$ by adding $k$-ary conjunction to the logical connectives. In particular, $\mathcal{B}$ is a complete subalgebra of $\mathcal{A}$. That is, if $X \subseteq B$ is such that $\sup X$ (the supremum of $X$ ) exists in $A$, then $\sup X$ exists in $B$, and they are equal, in symbols $\sum^{\mathcal{A}} X=\sum^{\mathcal{B}} X$.

1.4. $\mathcal{B}$ is not a neat reduct. Here we show that $\mathcal{B}$ is not a neat reduct. Recall that $\mathcal{B}$ was obtained from $\mathcal{A}$ by an infinite "cardinality twist", namely by keeping only countably many atoms below $1_{\text {Id }}$ and deleting the rest. In this section we show that first order logic cannot "see" this infinite cardinality twist. We start off by showing that the set of all elements in $B$ below $1_{02}$ remains countable.

FACT 1.4.1. The set $\left\{b \in B: b \leq 1_{02}\right\}$ is countable.

Proof. Let $Y=\left\{p(u, r): u \in{ }^{2} 3\right.$ and $\left.\operatorname{Rg} u \neq\{0,2\}\right\} \cup P_{\omega}(\langle 0,2\rangle) \cup$ $P_{\omega}(\langle 2,0\rangle)$. (Recall that $P_{\omega}\left(\left\langle u_{0}, u_{1}\right\rangle\right)=\left\{p\left(\left\langle u_{0}, u_{1}\right\rangle, r\right): r \in N\right\}$, where $N$ is a fixed countable subset of $R$.) Let $X=\left\{c_{i} a: i<2, a \in A\right\} \cup\left\{d_{01}\right\}$. Let $\mathcal{D}$ be the boolean subalgebra of $\mathcal{A}$ generated by $Y \cup X$. Then $D$ is a subuniverse of $\mathcal{A}$; the proof is similar to that of (2) in Fact 1.3.1 and so we omit it. Since $Y \subseteq D$, we have $B \subseteq D$ (in fact, $B=D$, but we do not need that much), and so $\mathrm{Rl}_{1_{02}} B \subseteq \mathrm{Rl}_{1_{02}} D$. It clearly suffices to show that $\left|\mathrm{Rl}_{1_{02}} D\right| \leq \omega$. To this end, let $\mathrm{rl}_{1_{02}}$ denote the following endomorphism of the structure $\langle A, \cup, \cap, \backslash\rangle$ :

$$
\mathrm{rl}_{1_{02}} a=a \cap 1_{02}, \quad a \in A .
$$


Then $\mathrm{Rl}_{1_{02}} D=\operatorname{Sg}^{\mathrm{Bl}(\mathcal{A})} \operatorname{rl}_{1_{02}}(X \cup Y)$. But $\mathrm{rl}_{1_{02}}(X \cup Y)$ is countable because $\left|P_{\omega}(\langle 0,2\rangle)\right|=\omega$ and $|X|<\omega$. Therefore $\mathrm{Rl}_{1_{02}} D$, generated as a boolean algebra by a countable set, is also countable.

Next we show that, were $\mathcal{B}$ a neat reduct, $\mathrm{Rl}_{1_{02}} B$ would be an uncountable set. This contradicts Fact 1.4.1. From this we infer that $\mathcal{B}$ is not a neat reduct. For this we need:

FACT 1.4.2. Let $\tau(x, y)$ denote the $S C_{2}$ term $c_{1}\left(c_{0} x \cap s_{1}^{0} c_{1} y\right) \cap c_{1} x \cap c_{0} y$. Let $\tau_{3}(x, y)$ denote the $S C_{3}$ term $c_{2}\left(s_{2}^{1} c_{2} x \cap s_{2}^{0} c_{2} y\right)$. Then:

(i) $\tau^{\mathcal{B}}\left(1_{01}, 1_{12}\right)=1_{02}$.

(ii) $S C_{3} \models \tau_{3}(x, y) \leq \tau\left(c_{2} x, c_{2} y\right)$.

Proof. (i) We have

$$
\tau^{\mathcal{B}}\left(1_{01}, 1_{12}\right)=c_{1}\left(c_{0} 1_{01} \cap s_{1}^{0} c_{1} 1_{12}\right) \cap c_{1} 1_{01} \cap c_{0} 1_{12} .
$$

We first compute $s_{1}^{0} c_{1} 1_{12}$ :

$$
\begin{aligned}
s_{1}^{0} c_{1} 1_{12} & =c_{0}\left(d_{01} \cap\left(1_{12} \cup 1_{11} \cup 1_{10}\right)\right)=c_{0}\left(d_{01} \cap 1_{11}\right) \\
& =c_{0} 1_{11} \quad(\text { by Fact } 1.3 .1(\mathrm{iv})) \\
& =c_{0} 1_{01} .
\end{aligned}
$$

Thus

$$
\tau^{\mathcal{B}}\left(1_{01}, 1_{12}\right)=c_{1}\left(c_{0} 1_{01} \cap c_{0} 1_{01}\right) \cap c_{1} 1_{01} \cap c_{0} 1_{12}=c_{1} 1_{01} \cap c_{0} 1_{12}=1_{02} .
$$

(ii) In our derivation we use the axiomatization given in Proposition 0.1. Now computing we get

$$
\begin{aligned}
\tau_{3}(x, y) & =c_{2}\left(s_{2}^{1} c_{2} x \cap s_{2}^{0} c_{2} y\right) \\
& \leq c_{2}\left(s_{2}^{1}\left(c_{0} c_{2} x \cap c_{1} c_{2} x\right) \cap s_{2}^{0}\left(c_{0} c_{2} y \cap c_{1} c_{2} y\right)\right) \\
& =c_{2}\left(s_{2}^{1} c_{0} c_{2} x \cap s_{2}^{1} c_{1} c_{2} x \cap s_{2}^{0} c_{0} c_{2} y \cap s_{2}^{0} c_{1} c_{2} y\right) \\
& \left.=c_{2}\left(s_{2}^{1} c_{0} c_{2} x \cap c_{1} c_{2} x \cap c_{0} c_{2} y \cap s_{2}^{0} c_{1} c_{2} y\right)\right) \\
& =c_{2}\left(s_{2}^{1} c_{0} c_{2} x \cap s_{2}^{0} c_{1} c_{2} y\right) \cap c_{1} c_{2} x \cap c_{0} c_{2} y \\
& =c_{2}\left(s_{2}^{1} c_{0} c_{2} x \cap s_{2}^{1} s_{1}^{0} c_{1} c_{2} y\right) \cap c_{1} c_{2} x \cap c_{0} c_{2} y \\
& =c_{2} s_{2}^{1}\left(c_{0} c_{2} x \cap s_{1}^{0} c_{1} c_{2} y\right) \cap c_{1} c_{2} x \cap c_{0} c_{2} y \\
& =c_{1} s_{1}^{2}\left(c_{0} c_{2} x \cap s_{1}^{0} c_{1} c_{2} y\right) \cap c_{1} c_{2} x \cap c_{0} c_{2} y \\
& =c_{1} s_{1}^{2} c_{2}\left(c_{0} c_{2} x \cap s_{1}^{0} c_{1} c_{2} y\right) \cap c_{1} c_{2} x \cap c_{0} c_{2} y \\
& =c_{1} c_{2}\left(c_{0} c_{2} x \cap s_{1}^{0} c_{1} c_{2} y\right) \cap c_{1} c_{2} x \cap c_{0} c_{2} y \\
& =c_{1}\left(c_{0} c_{2} x \cap s_{1}^{0} c_{1} c_{2} y\right) \cap c_{1} c_{2} x \cap c_{0} c_{2} y \\
& =\tau\left(c_{2} x, c_{2} y\right) .
\end{aligned}
$$

REMARK. $\tau_{3}(x, y)$ is more commonly denoted in the literature by $x ; y$. $x ; y$ abstracts composition of binary relations (cf. [16]). 
FACT 1.4.4. Let $\operatorname{Rd}_{S C} \mathcal{B}$ denote the $S C$ reduct of $\mathcal{B}$.

(i) Suppose that $\operatorname{Rd}_{S C} \mathcal{B} \subseteq \mathcal{N} r_{2}(\mathcal{C})$ with $\mathcal{C} \in S C_{3}$. Let

$$
D=\left\{\tau_{3}^{\mathcal{C}}(x, y): x, y \in N r_{2}(C), x \leq 1_{01}, y \leq 1_{12}\right\} .
$$

Then $|D|>\omega$. In fact, $P(\langle 0,2\rangle) \subseteq D$.

(ii) $\operatorname{Rd}_{S C} \mathcal{B} \notin N r_{2} S C_{3}$.

Proof. (i) Assume first that $\mathcal{C}$ is a set algebra. Then the result follows by noting that

$$
\tau_{3}^{\mathcal{C}}\left(p\left(\langle 0,1\rangle, r_{1}\right), p\left(\langle 1,2\rangle, r_{2}\right)\right)=p\left(\langle 0,2\rangle, r_{1}+r_{2}\right) .
$$

Now if $\mathcal{C}$ is not a set algebra, then replacing $\mathcal{C}$ by $\mathcal{E}=\operatorname{Sg}^{\mathcal{C}} B$, we deduce that $\mathcal{E}$ is isomorphic to a set algebra by $[22]\left({ }^{7}\right)$. This is so because $\mathcal{E}$ is an atomic algebra that is generated by a set of atoms, namely the set of atoms of $B$ that are functions. Indeed, every $p(u, r)$ for $u \in{ }^{2} 3$ and $r \in R$ is a binary relation that is a function. The same argument as above, but now applied to $\mathcal{E}$, works.

(ii) Assume, seeking a contradiction, that $\operatorname{Rd}_{S C} \mathcal{B}=\mathcal{N} r_{2} \mathcal{C}$, say, with $\mathcal{C} \in S C_{3}$. Let $x, y \in B$ be such that $x \leq 1_{01}$ and $y \leq 1_{12}$. Then $x$ and $y$ are 2-closed, i.e. $c_{2} x=x$ and $c_{2} y=y$. Also $\tau_{3}^{\mathcal{C}}(x, y) \in B$. Now

$$
\begin{aligned}
\tau_{3}^{\mathcal{C}}(x, y) & \leq \tau_{3}^{\mathcal{C}}\left(1_{01}, 1_{12}\right) \leq \tau^{\mathcal{C}}\left(c_{2} 1_{01}, c_{2} 1_{12}\right)=\tau^{\mathcal{B}}\left(1_{01}, 1_{12}\right) \\
& =1_{02} \quad(\text { by Fact } 1.4 .2(\mathrm{i})) .
\end{aligned}
$$

In other words we have

$$
D=\left\{\tau_{3}^{\mathcal{C}}(x, y): x \leq 1_{01}, y \leq 1_{12}\right\} \subseteq\left\{b \in B: b \leq 1_{02}\right\} .
$$

By Fact 1.4.1, $D$ must be countable. But this contradicts Fact 1.4.4(i). Thus, the proof of Theorem 1 is complete.

REMARK. We have actually proved the following: Let $\beta>2$. Let $L$ be any class such that $K s_{\beta}^{\text {reg }} \cap \operatorname{Lf} K_{\beta} \subseteq L \subseteq K_{\beta}$. Then $\mathbf{N} r_{2} L$ is not closed under forming elementary subalgebras, hence is not elementary. We conjecture that a slight modification of our construction can lift this result to higher dimensions. In this connection, [32] and [30] might be useful.

Acknowledgements. Thanks are due to an anonymous referee for careful reading and valuable suggestions. The author also wishes to thank Gábor Sági and Hajnal Andréka for fruitful discussions.

$\left({ }^{7}\right)$ Strictly speaking the result of Maddux and Tarski says that if $\mathcal{A}$ is an atomic relation algebra whose atoms are functions, then $A$ is representable. Unpublished work of Andréka and Givant generalizes this result to $C A$-like algebras of relations. For results of this kind-giving sufficient conditions for representability of atomic algebras of relations by imposing conditions on the atoms like density — we refer the reader to [4]. 


\section{References}

[1] M. Amer, Cylindric algebras of sentences, abstract, J. Symbolic Logic 58 (1993), 743.

[2] H. Andréka, Complexity of equations valid in algebras of relations, Ann. Pure Appl. Logic 89 (1997), 149-209.

[3] H. Andréka, T. Gergely and I. Németi, On universal algebraic constructions of logics, Studia Logica 36 (1977), 9-47.

[4] H. Andréka, S. Givant, S. Mikulás, I. Németi and A. Simon, Notions of density that imply representability in algebraic logic, Ann. Pure Appl. Logic 91 (1998), 93-190.

[5] H. Andréka, I. Németi and T. Sayed Ahmed, On neat reducts of algebras of logic, presented at Logic Colloquium 96; abstract appeared in Bull. Symbolic Logic.

[6] H. Andréka, A. Kurucz, I. Németi and I. Sain, General algebraic logic including algebraic model theory. An overview, in: Logic Colloquium '92, L. Csirmaz et al. (eds.), Stud. Logic Lang. Inform., CSLI Publ., 1995, 1-60.

[7] H. Andréka and I. Németi, Neat reducts of varieties, Studia Sci. Math. Hungar. 13 (1978), 47-51.

[8] H. Andréka and T. Sayed Ahmed, Omitting types in logics with finitely many variables, presented at Logic Colloquium 1998; abstract appeared in Bull. Symbolic Logic.

[9] - - - On omitting types, in preparation.

[10] W. J. Blok and D. Pigozzi, Algebraizable logics, Mem. Amer. Math. Soc. 396 (1989).

[11] C. C. Chang and H. J. Keisler, Model Theory, North-Holland, 1990.

[12] A. Diagneault and J. D. Monk, Representation theory for polyadic algebras, Fund. Math. 52 (1963), 151-176.

[13] M. Ferenczi, On representability of cylindric algebras, Abstracts Amer. Math. Soc. 13 (1992), 336.

[14] -, On representability of neatly embeddable cylindric algebras, J. Appl. NonClassical Logics, to appear.

[15] L. Henkin, J. D. Monk and A. Tarski, Cylindric Algebras, Part I, North-Holland, 1971.

[16] —, -, 一, Cylindric Algebras, Part II, North-Holland, 1985.

[17] L. Henkin, J. D. Monk, A. Tarski, H. Andréka and I. Németi, Cylindric Set Algebras, Lecture Notes in Math. 883, Springer, 1981.

[18] R. Hirsch, I. Hodkinson and R. Maddux, Relation algebra reducts of cylindric algebras and an application to proof theory, submitted.

[19] J. S. Johnson, Non-finitizability of classes of representable polyadic algebras, J. Symbolic Logic 34 (1969), 344-354.

[20] R. Maddux, Relation algebras and neat embeddability of cylindric algebras, Notices Amer. Math. Soc. 24 (1977), 298.

[21] - Topics in relation algebras, PhD dissertation, Univ. of California, Berkeley, 1978.

[22] R. Maddux and A. Tarski, A sufficient condition for the representability of relation algebras, Notices Amer. Math. Soc. 23 (1976), A-477.

[23] I. Németi, The class of neat reducts of cylindric algebras is not a variety but is closed w.r.t. HP, Notre Dame J. Formal Logic 24 (1983), 399-409.

[24] -, Algebraisation of quantifier logics, an introductory overview, preprint No 131996, Math. Inst., Hungarian Acad. Sci.; a shortened version appeared in Studia Logica 50 (1991), 465-569.

[25] D. Pigozzi, Amalgamation, congruence extension, and interpolation properties in algebras, Algebra Universalis 1 (1971), 269-349. 
[26] G. Sági, On the finitization problem of algebraic logic, $\mathrm{PhD}$ dissertation, Budapest, 1999.

[27] I. Sain, Searching for a finitisable algebraisation of first order logic, submitted.

[28] I. Sain and R. Thompson, Strictly finite schema axiomatization of quasi-polyadic algebras, in: H. Andréka et al. (eds.), Algebraic Logic, North-Holland, Amsterdam, 1991, 539-571.

[29] T. Sayed Ahmed, Algebras of sentences of logic, master's thesis, Cairo Univ., 1998.

[30] - , The class of neat reducts is not elementary, Logic J. IGPL 9 (2001), 31-65.

[31] - On amalgamation of reducts of polyadic algebras, Algebra Universalis, to appear.

[32] - A model theoretic solution to a problem of Tarski, Math. Logic Quart., to appear.

[33] - Amalgamation of algebras of logic, in preparation.

[34] T. Sayed Ahmed and I. Németi, On neat reducts of algebras of logic, Studia Logica 62 (2001), 229-269.

[35] A. Simon, What the finitization problem is not, in: Algebraic Methods in Logic and Computer Science, Banach Center Publ. 28, Inst. Math., Polish Acad. Sci., 1993, 95-116.

[36] A. Tarski and S. Givant, A Formalization of Set Theory without Variables, Colloq. Publ. 41, Amer. Math. Soc., 1987.

Department of Mathematics

Faculty of Science

Cairo University

Giza, Egypt

E-mail: rutahmed@rusys.eg.net

Received 27 February 2001;

in revised form 26 July 2001 